\title{
Enterotoxemia hemorrágica por Clostridium spp. en coexistencia con Sarcocystis spp. en alpacas (Vicugna pacos). Reporte de caso clínico
}

\author{
Sandoval, Patricio ${ }^{a}-$ Falconi, Mercy ${ }^{a}-$ Mena, Luis $^{b}$ \\ Cabrera, Nelson ${ }^{a}$ - Aponte, Pedro M. a,c* \\ a AGROCALIDAD, Agencia Ecuatoriana de Aseguramiento de la Calidad - AGROCALIDAD, \\ b Laboratorio de Diagnóstico Animal, km 14 1/2 Vía Interoceánica, La Granja, MAGAP, Tumbaco, Ecuador \\ AGROCALIDAD, Agencia Ecuatoriana de Aseguramiento de la Calidad - AGROCALIDAD, Sanidad Animal, \\ km 14 1/2 Vía Interoceánica, La Granja, MAGAP, Tumbaco, Ecuador \\ c SENESCYT, Secretaria Nacional de Educación Ciencia y Tecnología / Proyecto Prometeo

\section{RESUMEN}

La enterotoxemia hemorrágica es causada por espe-cies del género Clostridium. Estos afectan mayor-mente el tracto digestivo, vía exotoxinas. La sarco-cystiosis es producida por especies del género Sarco-cystis que produce quistes en el tejido muscular. El objetivo de este trabajo fue reportar un caso clínico inusual de ocurrencia simultánea de Clostridiosis y Sarcocistiosis en camélidos sudamericanos en el Ecuador. El estudio abarcó la caracterización clínica, necropsia, histopatología y microbiología en un caso de muerte súbita de una alpaca macho (Vicugna pacos) con antecedentes de emaciación, inapetencia, incoordinación y aislamiento. Los hallazgos macros-cópicos incluyeron edema gaseoso, con burbujas de gas en tejido subcutáneo, hemorragias intradérmicas difusas (abdomen y tórax), tracto digestivo vacio con abundante gas, peritonitis difusa, intestino delgado hiperémico con hemorragias petequiales generaliza-das y contenido sanguinolento, hígado cirrótico, riñones hemorrágicos, corazón agrandado, pericardi-tis, endocarditis, pulmones y mucosa traqueal hemo-rrágicos. Se evidenciaron quistes de Sarcocystis spp. en tejido muscular. Los hallazgos histopatológicos concordaron con los macroscópicos. El tejido hepático mostró abscesos y abundantes bacilos esporulados. Los músculos no presentaron bacilos, pero si miositis inflamatoria y quistes parasitarios. Numerosos bacilos Gram positivos esporulados presentes en hígado, pulmón y líquido peritoneal. En conjunto, los hallazgos sugieren: Enterotoxemia hemorrágica por Clostridium spp, conjuntamente con Sarcocystiosis crónica muscu-lar, esto último inusual (se evidencia normalmente en edades más avanzadas), condición posiblemente origi-nada por inmunosupresión previa asociada a estrés crónico. Se recomienda, en el caso de realizar nuevas importaciones de camélidos suramericanos, ubicar centros de acopio en ambientes agroecológicos similares al originario de la especie.

Palabras clave: Camélidos sudamericanos, Clostridio-sis, histopatología, microbiología, sarcocistiosis

\footnotetext{
* Correspondencia a: AGROCALIDAD, Laboratorio de Diagnóstico Animal, km 14 1/2 Vía Interoceánica, La Granja, MAGAP, Tumbaco, Ecuador. Teléfono: +593022372844 , ext 223.

Correo electrónico: apontep@gmail.com
} 